\title{
Insulin-like growth factor $1 /$ Child-Turcotte-Pugh composite score as a predictor of treatment outcomes in patients with advanced hepatocellular carcinoma treated with sorafenib
}

\author{
Yehia I. Mohamed ${ }^{1}$, Sunyoung Lee ${ }^{1}$, Lianchun Xiao $^{2}$, Manal M. Hassan ${ }^{3}$, Aliya \\ Qayyum4, Rikita Hiatia ${ }^{3}$, Roberto Carmagnani Pestana ${ }^{1}$, Abedul Haque ${ }^{5}$, Bhawana \\ George $^{5}$, Asif Rashid 6 , Dan G. Duda7, Hesham Elghazaly ${ }^{8}$, Robert A. Wolff ${ }^{1}$, Jeffrey \\ S. Morris ${ }^{2}$, James Yao ${ }^{1}$, Hesham M. Amin ${ }^{5}$ and Ahmed O. Kaseb ${ }^{1}$ \\ ${ }^{1}$ Department of Gastrointestinal Medical Oncology, The University of Texas MD Anderson Cancer Center, Houston, TX, USA \\ ${ }^{2}$ Department of Biostatistics, The University of Texas MD Anderson Cancer Center, Houston, TX, USA \\ ${ }^{3}$ Department of Epidemiology, The University of Texas MD Anderson Cancer Center, Houston, TX, USA \\ ${ }^{4}$ Department of Abdominal Imaging, The University of Texas MD Anderson Cancer Center, Houston, TX, USA \\ ${ }^{5}$ Department of Hematopathology, The University of Texas MD Anderson Cancer Center, Houston, TX, USA \\ ${ }^{6}$ Department of Pathology, The University of Texas MD Anderson Cancer Center, Houston, TX, USA \\ ${ }^{7}$ Steele Laboratories, Department of Radiation Oncology, Massachusetts General Hospital and Harvard Medical School, \\ Boston, MA, USA \\ ${ }^{8}$ Department of Clinical Oncology, Faculty of Medicine, Ain Shams University, Cairo, Egypt \\ Correspondence to: Ahmed O. Kaseb, email: akaseb@mdanderson.org \\ Keywords: IGF-1; Child-Pugh; sorafenib; liver reserve; hepatocellular carcinoma
}

Received: November 09, $2020 \quad$ Accepted: March 08, $2021 \quad$ Published: April 13, 2021

Copyright: ( 2021 Mohamed et al. This is an open access article distributed under the terms of the Creative Commons Attribution License (CC BY 3.0), which permits unrestricted use, distribution, and reproduction in any medium, provided the original author and source are credited.

\section{ABSTRACT}

Background: Sorafenib was the first systemic therapy approved for the treatment of Child-Turcotte-Pugh (CTP) class A patients with advanced hepatocellular carcinoma (HCC). However, there are no biomarkers to predict survival and treatment outcomes and guide HCC systemic therapy. Type 1 insulin-like growth factor (IGF-1)/CTP composite score has emerged as a potential hepatic reserve assessment tool. Our study investigated the association of the IGF/CTP score with overall survival (OS) and progression-free survival (PFS) of HCC patients treated with sorafenib.

Materials and Methods: In this prospective study, patients with HCC were treated with sorafenib and followed up until progression/death. We calculated the IGF/CTP score and used the Kaplan-Meier method and log-rank test to estimate and compare the time-to-event outcomes between patient subgroups.

Results: 171 patients were included, 116 of whom were CTP class A. Median PFS for IGF/CTP score AA and AB patients were 6.88 and 4.28 months, respectively $(p=0.1359)$. Median OS for IGF/CTP score AA and AB patients were 14.54 and 7.60 months, respectively $(p=0.1378)$. The PFS and OS was superior in AA patients, but the difference was not significant, likely due to the sample size. However, there was a significant difference in early OS and PFS curves between AA and AB $(p=0.0383$ and $p=0.0099$ ), respectively.

Conclusions: In CTP class A patients, IGF/CTP score B was associated with shorter PFS and OS, however, study was underpowered to reach statistical significance. If validated in larger cohorts, IGF/CTP score may serve as stratification tool in clinical trials, a hepatic reserve assessment tool for HCC outcomes prediction and to assist in therapy decisions. 


\section{INTRODUCTION}

Accurate assessment of the functional hepatic reserve is important to the prognostic and treatment prediction for patients with liver disease $[1,2]$. The Child-TurcottePugh (CTP) qualitative scoring system is used to assess severity of cirrhosis, survival prospects hepatic reserve, guiding treatment decisions, and stratifying patients with hepatocellular carcinoma ( $\mathrm{HCC}$ ) for clinical trial entry; It classifies patients with liver disease into 3 groups (A, B, and $\mathrm{C}$ ), this classification is based on bilirubin and albumin levels, prothrombin time, and subjective assessments of encephalopathy and ascites $[1,2]$.

Several prospective and retrospective studies have confirmed that HCC patients in CTP classes B and C showed worse prognoses and accelerated decline in liver function as compared with CTP A patients $[3,4]$. Accordingly, clinical trials studying systemic therapies for HCC; include only those with CTP A classification, to assess the effects of the systemic therapy under investigation without the confounding issues of hepatic failure or death which may result from an underlying poor liver reserves [3-5]. However, it is known now that the clinical outcomes for patients can vary even within those in same CTP class, including class A. In addition, subjective variables, ascites and encephalopathy of the CTP scoring system have been considered its major shortcoming because they are difficult to grade, vary daily, and could be affected by symptomatic management [6-11].

The majority of circulating insulin-like growth factor (IGF) is synthesized and secreted by the liver [12]. Therefore, circulating IGF-1 level has been shown to decrease dramatically in Chronic liver disease and HCC [13-18]; We recently reported a significant correlation between advanced clinicopathologic parameters and low IGF-1 levels HCC patients [19, 20]. Subsequently, we prospectively validated a revised CTP scoring system by replacing the subjective clinical assessment of ascites and encephalopathy in the CTP score with objectively quantified plasma IGF-1 levels to create new IGF/CTP score classes (IGF/CTP-A, -B, and -C, with A indicating low risk, $\mathrm{B}$ indicating intermediate risk, and $\mathrm{C}$ indicating high risk). This new scoring system was significantly associated with HCC survival prediction [21-23]. In addition, our results showed that a significant number of patients in the old CTP class A were reassigned as IGF/ CTP-B or -C and had significantly poorer survival than did those in IGF/CTP-A, proving the usefulness of the IGF/ CTP composite score to refine the CTP scoring system's prognostic accuracy. Our previously published work incorporating IGF-1 into BCLC and CLIP scores showed higher prognostic value [24], and our recently published paper incorporated IGF-1 into MELD score, which significantly improved the score's ability to predict OS in 2 independent cohorts of HCC patients [25]. However, we did not assess its predictive ability in patients treated with systemic therapy. Sorafenib, the first drug approved for the treatment of HCC, was approved after randomized, placebo-controlled trials demonstrated that it improved the overall survival (OS) of CTP class A patients with advanced HCC [26, 27]; the purpose of our current study was to investigate the usefulness of the IGF/CTP score in predicting overall survival (OS) and progression-free survival (PFS) in CTP class A patients with advanced HCC who were treated with sorafenib. Our overall hypothesis was that the IGF/CTP score could divide CTP A patients into more refined subgroups on the basis of OS and PFS.

\section{RESULTS}

\section{Patient characteristics}

A total of 171 patients from MD Anderson meeting our criteria were screened and included into our study. Baseline characteristics (Table 1); Most patients (98\%) were classified under the Barcelona Clinic Liver Cancer (BCLC) stage C. Notably, $75 \%$ of the patients were 70 years or younger at the time of diagnosis, and $77.2 \%$ were males. During the follow-up period, 100 patients died. The median OS was 11.48 months (95\% CI, 8.75-15.33 months; Supplementary Table 1) and the median followup time was 19.9 months (95\% CI, 14.5-33.8 months). One hundred twenty-two patients had disease progression on sorafenib or died. The estimated median PFS was 5.30 months (95\% CI, 4.70-6.84 months; Supplementary Table 1). The estimated 1-year failure-free probability was $19 \%$ (95\% CI, 0.13-0.27).

\section{Circulating IGF-1 is associated with survival in sorafenib-treated patients}

OS and PFS significantly differed between groups of patients when stratified by IGF-1 levels. Compared to patients with high IGF-1 levels (> $26.0 \mathrm{ng} / \mathrm{mL}$ ), patients with low IGF-1 levels $(\leq 26.0 \mathrm{ng} / \mathrm{mL}$ ) had shorter median OS durations (12.83 months [95\% CI, 10.62-20.62 months] vs. 5.23 months [95\% CI, 4.28-10.66 months], respectively; $p=0.003$ ) and shorter median PFS durations (6.05 months [ $95 \%$ CI, 4.97-9.41 months] vs. 4.18 months [95\% CI, 2.99-5.23 months], respectively; $p=0.02$ ).

\section{IGF/CTP score is associated with survival in sorafenib-treated patients}

Both the CTP and the IGF/CTP scores classified patients into three risk groups that differed significantly in terms of OS and PFS; low (A)-, intermediate (B)-, and high (C). After IGF/CTP scoring, 87 of 116 CTP class A patients were reclassified as IGF/CTP-A (AA) and 29 patients were reclassified as IGF/CTP-B (AB) (Supplementary Table 1). Seven of 55 CTP class B patients were reclassified as IGF/CTP-A (BA), 35 were 
Table 1: Baseline clinical characteristics of 171 patients with hepatocellular carcinoma treated with sorafenib

\begin{tabular}{|c|c|}
\hline Variable & No. of patients (\%) \\
\hline \multicolumn{2}{|l|}{ Age, years } \\
\hline$\leq 40$ & $2(1.2)$ \\
\hline $41-50$ & $7(4.1)$ \\
\hline $51-60$ & $48(28.1)$ \\
\hline $61-70$ & $71(41.5)$ \\
\hline$>70$ & $43(25.1)$ \\
\hline \multicolumn{2}{|l|}{ Diabetes } \\
\hline No & $95(55.6)$ \\
\hline Yes & $71(41.5)$ \\
\hline Unknown & $5(2.9)$ \\
\hline \multicolumn{2}{|l|}{ Sex } \\
\hline Female & $39(22.8)$ \\
\hline Male & $132(77.2)$ \\
\hline \multicolumn{2}{|c|}{ History of drinking alcohol } \\
\hline No & $85(49.7)$ \\
\hline Yes & $75(43.9)$ \\
\hline Unknown & $11(6.4)$ \\
\hline \multicolumn{2}{|c|}{ Family history of liver cancer } \\
\hline No & $136(79.5)$ \\
\hline Yes & $7(4.1)$ \\
\hline Unknown & $28(16.4)$ \\
\hline \multicolumn{2}{|c|}{ Personal History of non-HCC cancer } \\
\hline No & $136(79.5)$ \\
\hline Yes & $28(16.4)$ \\
\hline Unknown & $7(4.1)$ \\
\hline \multicolumn{2}{|l|}{ Race } \\
\hline Asian & $2(1.2)$ \\
\hline Black & $24(14.0)$ \\
\hline Hispanic & $9(5.3)$ \\
\hline White & $129(75.4)$ \\
\hline Unknown & $7(4.1)$ \\
\hline \multicolumn{2}{|c|}{ History of tobacco use } \\
\hline No & $56(32.7)$ \\
\hline Yes & $104(60.8)$ \\
\hline Unknown & $11(6.4)$ \\
\hline \multicolumn{2}{|l|}{ Ascites } \\
\hline Moderate & $21(12.3)$ \\
\hline None & $119(69.6)$ \\
\hline Slight & $31(18.1)$ \\
\hline \multicolumn{2}{|c|}{ Evidence of cirrhosis } \\
\hline No & 37 (21.6) \\
\hline
\end{tabular}


CTP class

Metastasis

No

Yes

Unknown

Tumor nodularity

Multinodular

Uninodular

Vascular invasion

No

Yes

Unknown

Lymph node metastasis

No

Yes

Unknown

BCLC stage

A

B

Abbreviations: BCLC, Barcelona Clinic Liver Cancer; CTP, Child-Turcotte-Pugh; ECOG, Eastern Cooperative Oncology Group.

reclassified as IGF/CTP-B (BB), and 13 were reclassified as IGF/CTP-C (BC).

Among the patients classified as CTP class A, the median OS duration was 14.54 months for those reclassified as AA and 7.60 months for those reclassified as $\mathrm{AB}(p=0.13)$; although the OS difference favored the AA patients, the $p$ value was not significant. The hazard ratio (HR) for AA vs. AB patients was 1.49, and our study power calculation suggested that 325 participants with 220 events would be needed to achieve the $80 \%$ power needed to detect this HR, although the current data had only $39 \%$ power. We used the Wald test to compare OS probabilities between $\mathrm{AA}$ and $\mathrm{AB}$ patients at 7.60 months, 12.00 months, and 14.54 months (Table 2). The analysis indicated that AA patients had a significantly higher OS probability at 7.60 months (Wald test $p$ value $=0.030$ ) and at 12.00 months (Wald test $p$ value $=0.046$ ). At 14.54 months, the OS probability was higher in AA patients but not statistically significant (Wald test $p$ value $=0.49$ ). In addition, the log-rank test was used to compare OS curves censored at 12 months, i.e., patients who survived longer than 12.00 monthswere censored at 12.00 months. The log-rank test indicated that AA patients had significantly longer OS than $\mathrm{AB}$ patients ( $p=0.038$ ) (Figure 1).

The median PFS time was 6.88 months for AA patients and 4.28 months for $\mathrm{AB}$ patients $(p=0.1378)$, and although the PFS difference favored the AA patients, the $p$ value was not significant. However, our study was underpowered to detect statistically significant differences in the median PFS because of its small sample size. We used the Wald test to compare the PFS probabilities between $\mathrm{AA}$ and $\mathrm{AB}$ patients at 4.28 months, 5.00 months, 6.00 months, 6.88 months, and 8.00 months (Table 3). The analysis indicated that the AA patients had a significantly higher OS probability at 4.28 months (Wald test $p$ value $=0.012)$, at 5.00 months (Wald test $p$ value $=0.043)$, at 
Table 2: Comparison of $O S$ probabilities for $A A$ and $A B$ patients at 7.60, 12.00, and 14.54 months

\begin{tabular}{lcc}
\hline IGF/CTP classification & OS probability at $\mathbf{7 . 6 0}$ months $\mathbf{( 9 5 \%} \mathbf{C I})$ & Wald test $\boldsymbol{p}$ value \\
\hline $\mathrm{AA}$ & $0.73(0.63,0.84)$ & 0.0296 \\
$\mathrm{AB}$ & $0.48(0.32,0.72)$ & \\
& OS probability at $\mathbf{1 2 . 0 0}$ months $(\mathbf{9 5 \%} \mathbf{C I})$ & Wald test $\boldsymbol{p}$ value \\
$\mathrm{AA}$ & $0.62(0.51,0.75)$ & 0.046 \\
$\mathrm{AB}$ & $0.38(0.23,0.64)$ & Wald test $\boldsymbol{p}$ value \\
& OS probability at $\mathbf{1 4 . 5 4}$ months $(\mathbf{9 5 \%} \mathbf{C I})$ & 0.4915 \\
$\mathrm{AA}$ & $0.49(0.37,0.63)$ & \\
AB & $0.38(0.23,0.64)$ & \\
\hline
\end{tabular}

Abbreviations: CTP, Child-Turcotte-Pugh; IGF, insulin-like growth factor-1; OS, overall survival.

6.00 months (Wald test $p$ value $=0.036$ ), at 6.88 months (Wald test $p$ value $=0.017$ ), and at 8.00 months (Wald test $p$ value $=0.017)$. The PFS probability was higher in the AA patients at 10.00 and 12.00 months (Wald test $p$ values $=0.14$ and 0.25 , respectively), but the differences were not statistically significant. In addition, the log-rank test was used to compare PFS curves censored at 8 months, i.e., patients who survived longer than 8.00 months without disease progression were censored at 8.00 months. The log-rank test indicated that AA patients had significantly better early PFS than AB patients $(p=0.0099)$ (Figure 2$)$.

OS and PFS results for the CTP class A patients by plasma IGF-1 levels are summarized in Supplementary Table 2. Patients with low IGF-1 levels $(\leq 26.0 \mathrm{ng} / \mathrm{mL})$ had worse OS and PFS than those with higher IGF-1 levels $(>26.0 \mathrm{ng} / \mathrm{mL})$, but these differences were not statistically significant.

OS and PFS results for the CTP class B patients by plasma IGF-1 levels are summarized in Supplementary Table 3. Patients with low IGF-1 $(\leq 26.0 \mathrm{ng} / \mathrm{mL})$ had significantly worse OS and PFS than those with higher IGF-1 levels $(>26.0 \mathrm{ng} / \mathrm{mL})(p=0.0027$ and $p=0.028$ respectively)and they also had worse PFS.

OS and PFS results for the CTP class B patients by IGF/CTP scores (Supplementary Table 3). Of the 55 patients classified as CTP class B, 35 were reclassified as IGF/CTP-B (BB) and had a median OS of 7.07 months (95\% CI, 3.98-21.58 months) and a median PFS of 4.54 months (95\% CI, 3.82-10.26 months). Seven CTP class B patients were reclassified as IGF/CTP-A (BA) and had a median OS of 6.71 months $(95 \%$ CI, 4.41 months-not applicable [N/A]) and a median PFS of 1.97 months (95\% CI, 1.84 months-N/A). Thirteen CTP class B patients were reclassified as IGF/CTP-C (BC) and had a median OS of 4.18 (95\% CI, 2.07 months-N/A) and a median PFS of 3.29 months ( $95 \%$ CI, 2.07 months-N/A).

To evaluate whether IGF-1 provides additional prognostic effects on OS and PFS to CTP in this dataset, Cox models were fitted to include both CTP and IGF-1. The analysis showed that IGF-1 provided additional prognostic effects $(\mathrm{HR}=1.87$ and $p=0.0054)$ for $\mathrm{OS},(\mathrm{HR}=1.59$ and $p=0.0319$ ) for PFS. (Supplementary Table 1). To assess the performance of IGF-modified-CTP and CTP in their predictive capacity of OS and PFS, Harrell's C-index was calculated for each of score systems, and U statistics was used to compare the c-indices between the two score systems. C-index for IGF-CTP was slightly higher than for CTP, but it was not statistically significant ( $p=0.61$ for OS and $p=0.96$ for PFS, respectively, Table 4).

$\mathrm{C}$-index was calculated to assess the predictive capacity of IGF-modified-CTP for OS and PFS in CTP A patients. In addition, Cox models including albumin + bilirubin + INR with and without IGF-1 were fitted, and $\mathrm{C}$-index was calculated for each model. The analysis showed that $\mathrm{C}$-index for OS was statistically significant higher in the model with IGF-1 than that in the model without IGF-1 ( $p=0.026$, Table 4). The C-index for PFS was slightly higher in the model with IGF-1 than that in the model without IGF-1, but it was not statistically significant $(p=0.11)$ (Table 4).

In patients with CTP class A HCC, we compared the rate of adverse events among those reclassified as IGF/CTP-A (AA) to that of patients reclassified as IGF/ CTP-B (AB) (Supplementary Table 4). There was a higher overall rate of adverse events in the AA patients than in the $\mathrm{AB}$ patients, especially in regard to grade III-IV adverse events, bleeding in the upper and lower gastrointestinal tract, nose bleeding, renal failure, liver failure, encephalopathy, fatigue, weight loss, anorexia, and vomiting.

\section{DISCUSSION}

This study supports our hypothesis that the IGF/CTP score can distinguish and refine CTP class A patients. We found that patients who were reclassified as IGF/CTP-B or -C had poorer prognoses (shorter OS and PFS durations than those reclassified as IGF/CTP-A). However, for CTP class A patients, the threshold for statistical significance for the OS and PFS durations of the reclassified groups $\mathrm{AA}$ and $\mathrm{AB}$ were not met because of the limited power of the study (39\%); our power calculation suggested that 325 participants with 220 events would be needed to achieve 
Table 3: Comparison of PFS probabilities for $\mathrm{AA}$ and $\mathrm{AB}$ patients at 4.28, 5.00, 6.00, 6.88, and 8.00 months

\begin{tabular}{|c|c|c|}
\hline IGF/CTP classification & PFS probability at 4.28 months $(95 \% \mathrm{CI})$ & Wald test $p$ value \\
\hline $\mathrm{AA}$ & $0.75(0.66,0.86)$ & 0.0122 \\
\hline \multirow[t]{2}{*}{$\mathrm{AB}$} & $0.48(0.33,0.71)$ & \\
\hline & PFS probability at 5.00 months $(95 \% \mathrm{CI})$ & Wald test $p$ value \\
\hline $\mathrm{AA}$ & $0.67(0.57,0.78)$ & 0.0431 \\
\hline \multirow[t]{2}{*}{$\mathrm{AB}$} & $0.44(0.29,0.68)$ & \\
\hline & PFS probability at 6.00 months $(95 \%$ CI) & Wald test $p$ value \\
\hline AA & $0.55(0.44,0.68)$ & 0.0364 \\
\hline \multirow[t]{2}{*}{$\mathrm{AB}$} & $0.32(0.18,0.56)$ & \\
\hline & PFS probability at 6.88 months $(95 \% \mathrm{CI})$ & Wald test $p$ value \\
\hline AA & $0.49(0.38,0.62)$ & 0.0171 \\
\hline \multirow[t]{2}{*}{$\mathrm{AB}$} & $0.24(0.12,0.48)$ & \\
\hline & PFS probability at 8.00 months $(95 \% \mathrm{CI})$ & Wald test $p$ value \\
\hline $\mathrm{AA}$ & $0.49(0.38,0.62)$ & 0.0171 \\
\hline $\mathrm{AB}$ & $0.24(0.12,0.48)$ & \\
\hline
\end{tabular}

Abbreviations: CTP, Child-Turcotte-Pugh; IGF, insulin-like growth factor-1; PFS, progression-free survival.

the $80 \%$ power needed to detect significant difference in survival outcome.

Assessing liver reserve in $\mathrm{HCC}$ is of a great value as a tool for stratification of patients in clinical trials as well as to predict HCC outcome and guide therapy decisions in routine practice [28]; Sorafenib, the first drug approved for the treatment of HCC, was approved after randomized, placebo-controlled trials demonstrated that it improved the overall survival (OS) of CTP class A patients with advanced HCC [26, 27] with high importance to

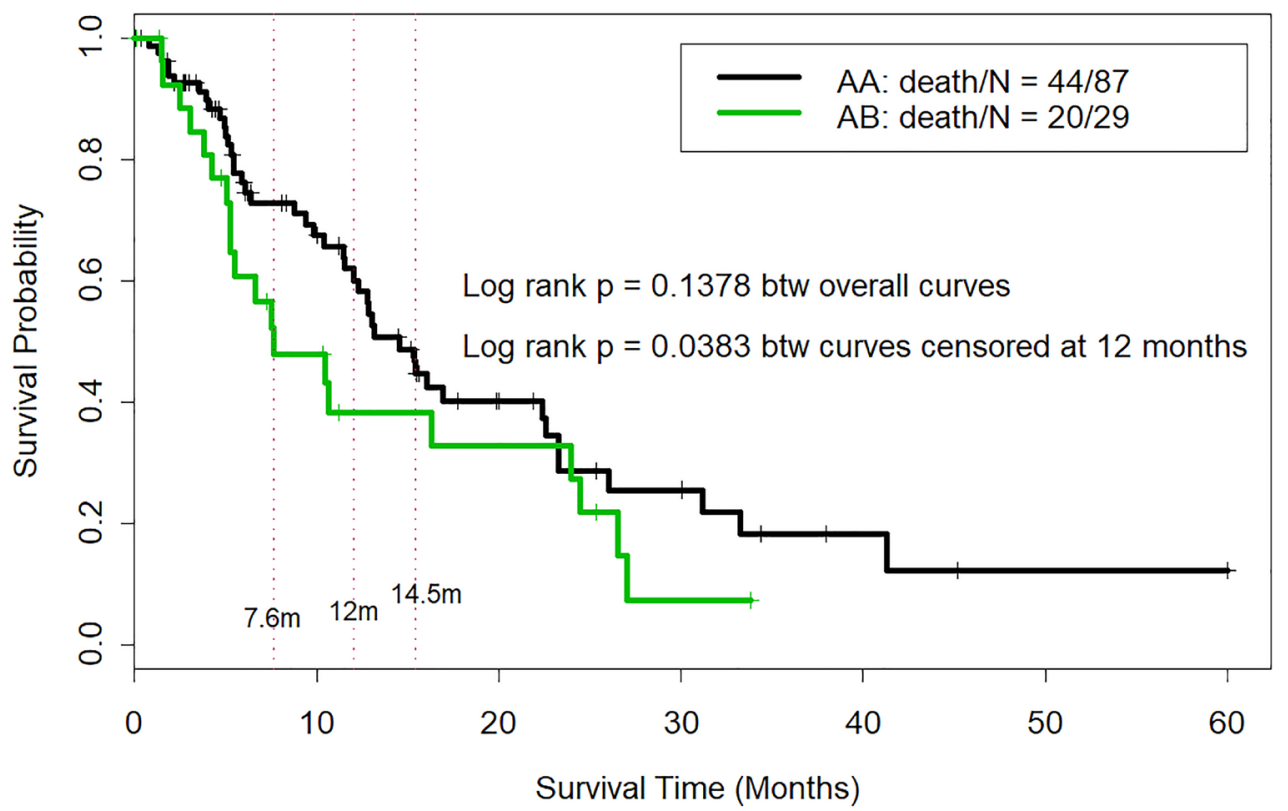

No. at Risk

$\begin{array}{llllllll}\mathrm{AA} & 87 & 38 & 16 & 8 & 3 & 1 & 1 \\ \mathrm{AB} & 29 & 11 & 6 & 1 & 0 & 0 & 0\end{array}$

Figure 1: Comparison of overall survival in CTP class A patients who were reclassified as IGF/CTP-A (AA) and or IGF/CTP-B (AB). -Log-rank test was used to compare OS curves among patients who survived longer than 12.00 months; these patients were censored at 12.00 months. The log-rank test indicated that AA patients had significantly longer OS than AB patients $(p=0.038)$. 
Table 4: Estimated C-index for CTP, IGF-modified-CTP in all patients and in CTP A patients, as well as for Cox models with and without IGF-1 in CTP A patients

\begin{tabular}{llcc}
\hline All patients & & C Index (95\% CI) & P value \\
\hline OS & CTP & $0.58(0.53,0.63)$ & 0.61 \\
& IGF-CTP & $0.60(0.54,0.66)$ & \\
PFS & CTP & $0.56(0.52,0.61)$ & 0.96 \\
& IGF-CTP & $0.57(0.52,0.62)$ & \\
CTP A patients & & & \\
OS & IGFCPT & $0.55(0.49,0.61)$ & \\
PFS & IGFCPT & $0.56(0.51,0.61)$ & \\
OS & Model with albumin + bilirubin + INR & $0.64(0.56,0.73)$ & 0.026 \\
& Model with albumin + bilirubin + INR + IGF1 & $0.65(0.57,0.74)$ & \\
PFS & Model with albumin + bilirubin + INR & $0.61(0.54,0.68)$ & 0.11 \\
& Model with albumin + bilirubin + INR + IGF1 & $0.63(0.55,0.70)$ & \\
\hline
\end{tabular}

select which patients will profit the most from sorafenib; especially that systemic therapy in HCC carries high cost which adds a significant burden to health care system budgets; thus particularly important to identify which HCC patients will benefit from systemic therapy as distinct from those in whom systemic therapy will pose lower survival benefit. In this context, applying a simple, noninvasive, routine laboratory test, relatively low cost marker for liver reserve assessment will be helpful.

Our group recently reported a significant correlation between advanced clinicopathologic parameters and lower plasma IGF-1 levels in patients with HCC, IGF-1 has emerged as a substitute biomarker for Liver reserve and we have showed the usefulness of adding it to the CTP score; substituting ascites and encephalopathy [19]. This led to our development and prospective validation of our revised and improved IGF/CTP scoring system, which replaced the subjective clinical assessment of ascites and encephalopathy included in the original CTP scoring system with objectively quantified plasma IGF1 levels and created new IGF/CTP score classes (A-C). The IGF/CTP score is advantageous not only because it uses only objective laboratory variables that are routinely assessed in clinical practice, but also because it includes

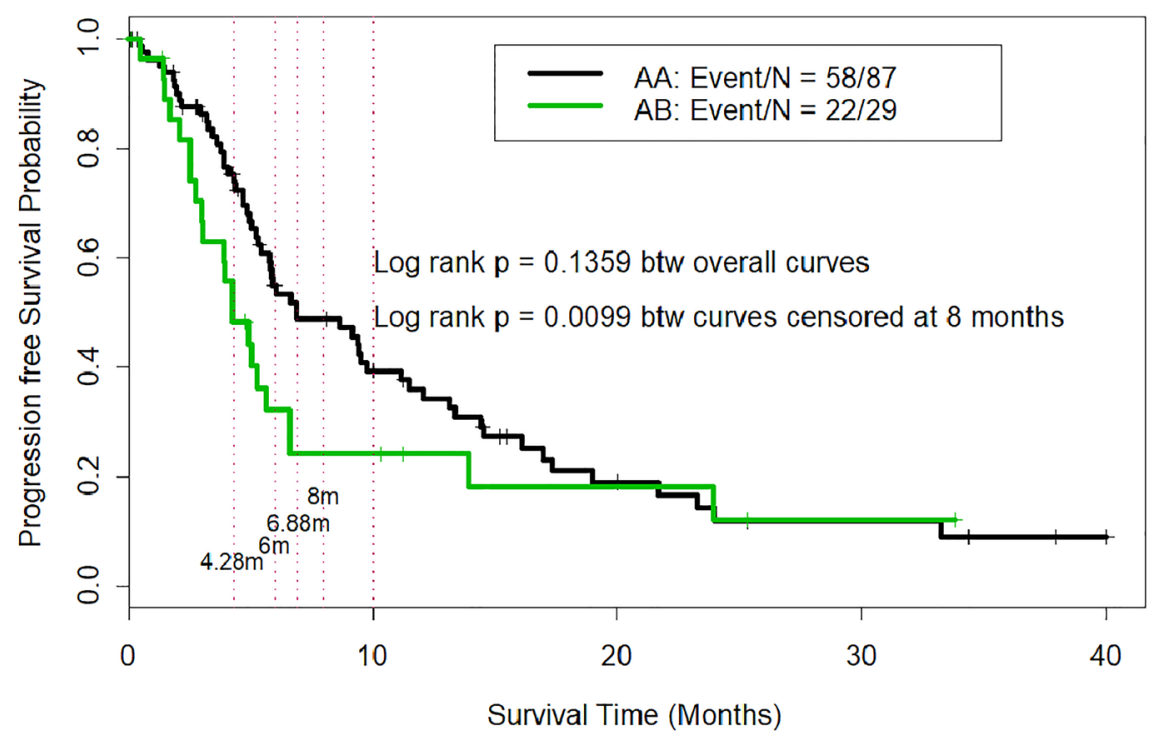

No. at Risk

$\begin{array}{llclll}\mathrm{AA} & 87 & 25 & 9 & 4 & 1 \\ \mathrm{AB} & 29 & 6 & 3 & 1 & 0\end{array}$

Figure 2: Comparison of progression-free survival in CTP class A patients who were reclassified as IGF/CTP-A (AA) and or IGF/CTP-B (AB). -Log-rank test was used to compare PFS curves among patients who survived longer than 8.00 months without disease progression; these patients were censored at 8.00 months. The log-rank test indicated that AA patients had significantly better early PFS than AB patients $(p=0.0099)$. 


\begin{tabular}{lccc}
\hline & \multicolumn{3}{c}{} \\
\cline { 2 - 4 } Variable & $\mathbf{1}$ & $\mathbf{2}$ & $\mathbf{3}$ \\
\hline Encephalopathy & None & Mild (1-2) & Severe (3-4) \\
Ascites & None & Mild/moderate & Severe/refractory \\
Albumin, g/dL & $>3.5$ & $2.8-3.5$ & $<2.8$ \\
PT, s & $<4$ & $4-6$ & $>6$ \\
Bilirubin, $\mathrm{mg} / \mathrm{dL}+$ & $<2$ & $2-3$ & $>3$ \\
IGF-1, ng/mL & $>50$ & $26-50$ & $<26$ \\
\hline
\end{tabular}

IGF/CTP score classes: A, 4-5 points; B, 6-7 points; C, more than 7 points. ${ }^{ \pm}$In patients with primary biliary cirrhosis and primary sclerosing cholangitis, the upper limit of bilirubin for 1 point is $4 \mathrm{mg} / \mathrm{dL}$ and the upper limit for 2 points is $10 \mathrm{mg} / \mathrm{dL}$. Abbreviations: CTP, Child-Turcotte-Pugh; IGF-1, type 1 insulin-like growth factor; PT, prothrombin time.

the plasma IGF-1 level, which is a standardized, Clinical Laboratory Improvement Amendments-certified test and therefore ready for implementation in routine practice. Our prior published data showed that the IGF/CTP scoring system significantly improved the prediction of survival in HCC patients [21]. Moreover, our previous results demonstrated that a significant number of patients with CTP class A were reclassified as IGF/CTP-B and $-\mathrm{C}$ and had significantly poorer survival than those who were reclassified as IGF/CTP-A. However, we had not previously assessed the outcomes of sorafenib-treated patients reclassified according to our new scoring system.

Our study is the first prospective validation of the IGF/CTP scoring system association with the outcomes among patients with $\mathrm{HCC}$ treated with sorafenib. Although our study was not powered to determine the predictive value of the IGF/CTP score in regard to median OS and PFS durations in CTP class A patients treated with sorafenib, our subset analyses of OS and PFS at different timepoints were statistically significant and, if independently validated, could change the standard approach to assessing hepatic reserve in patients with $\mathrm{HCC}$.

Our current study included an exploratory analysis of 55 CTP class B patients with HCC. This is an important population to study, as most clinical trials of systemic therapies for HCC include only CTP class A patients. In our current study, CTP class B patients with HCC who were reclassified as IGF/CTP-A (BA) had OS and PFS durations of 6.71 months and 1.97 months, respectively, while those reclassified to IGF/CTP-B (BB) had OS and PFS durations of 7.07 months and 4.54 months, respectively. These findings did not reach the statistical threshold for significance because of the small number of CTP class B patients in the current study. However, our exploratory analysis of the CTP class B group based only on IGF-1 levels support the hypothesis that IGF-1 levels predict survival outcomes in patients with HCC. The analysis indicated that patients with IGF-1 levels greater than $26.0 \mathrm{ng} / \mathrm{mL}$ had significantly longer OS and PFS durations than patients with IGF levels less than or equal to $26.0 \mathrm{ng} / \mathrm{mL}(p=0.0027)$. Nevertheless, these findings remain exploratory due to the small number of CTP class B patients in our study and the retrospective nature of the analysis. Therefore, larger studies of IGF-1 levels in this population are needed.

One of the limitations of our study is that it was a single-center study in a selected population. Second, it had insufficient power to reach statistical significance, however it showed a clear trend in better survival outcomes in AA patients as compared to $\mathrm{AB}$ as aforementioned. To validate the observations in the current study, a larger sample size will be required. However, we believe that there is critical need for future large randomized studies with enough power to confirm our results and validate the utility of IGF1 as a predictive score for systemic therapy response.

In conclusion, our results demonstrate that, in CTP class A patients with advanced HCC treated with sorafenib, the IGF/CTP scoring system provides more accurate associations with survival than the CTP score. This finding should be validated in studies with larger sample sizes. If our results are validated in independent future studies, our approach of computing CTP scores using IGF-1 levels and other laboratory-based parameters that are less subjective than the clinical assessments currently used may lead to a paradigm shift in predicting the efficacy and toxicity of systemic HCC therapies and in stratifying patients in $\mathrm{HCC}$ clinical trials. Our approach could also help differentiate between CTP class A patients who may benefit from active therapy and those in whom active therapy should be deferred to avoid unnecessary harm and save health care resources.

\section{MATERIALS AND METHODS}

\section{Study design and study population}

The University of Texas MD Anderson Cancer Center's Institutional Review Board approved this prospective study, and informed consent was obtained from all enrolled patients. Our study was prospectively designed to collect pretreatment samples from sorafenib patients, follow them until progression, and analyze correlation with 
pretreatment IGF-1 score. Therefore, score analysis was done after therapy was concluded for all patients. Adult patients with pathologically or radiologically confirmed HCC, as defined by the American Association for the Study of Liver Diseases, who were treated at MD Anderson Cancer Center from January 2004 to July 2018 were enrolled in the study. Patients' blood samples and epidemiologic and clinical data were collected, and plasma samples were analyzed retrospectively for IGF-1. Peripheral venous blood specimens $(3-5 \mathrm{~mL})$ were collected from all patients; anticoagulated with ethylenediaminetetraacetic acid, and centrifuged for 15 minutes at $3000 \mathrm{rpm}$. The plasma was then removed, aliquoted, and snap frozen at $-20^{\circ} \mathrm{C}$. Baseline IGF-1 levels, clinical characteristics, Adverse events to treatment using CTCAE V4.03; and epidemiological data were retrieved from the medical records. IGF/CTP scores were calculated and patients were classified as IGF/CTP-A, $-\mathrm{B}$, or $-\mathrm{C}$ based on their serum bilirubin and albumin levels, prothrombin times, and plasma IGF-1 levels $(\leq 26 \mathrm{ng} / \mathrm{ml}$ : score of $3,>26$ and $\leq 50$, score of 2 and score of 1 for IGF $>50$ ); IGF-CTP scores have a range between 4-12; 4-5 for IGF-CTP(A), 6-7 for IGF-CTP (B) and > 7 for IGF-CTP (C) (Table 5); PFS was calculated from the date that sorafenib treatment began to the date of disease progression or death. OS was calculated from the date that sorafenib treatment began to the date of death or of the last follow-up.

Frequencies and percentages were used to tabulate patients' baseline characteristics and adverse events. The Fisher exact test was used to compare the occurrence of adverse events between subgroups of patients. The Kaplan-Meier method was used to estimate time-to-event outcomes (e.g., OS, PFS), and the log-rank test and Cox proportional hazards models was used to compare the time-to-event outcomes. In addition, we used the Wald test to compare OS and PFS probabilities between AA and $\mathrm{AB}$ patients at some early survival time points; Harrell's concordance index (c-index) and U statistics [29] were used to evaluate the predictive capacity between CTP and IGF-CTP score systems and between Cox models with and without IGF-1. A $p$ value if less than 0.05 was considered statistically significant. S+ version 8.2.0 (TIBCO Software Inc., Palo Alto, CA, USA) and SAS version 9.4 (SAS Institute, Cary, NC, USA) were used for the analyses.

\section{Abbreviations}

CTP: Child-Turcotte-Pugh; HCC: hepatocellular carcinoma; HR: hazard ratio; IGF-1: type 1 insulin-like growth factor; N/A: not applicable; OS: overall survival; PFS: progression-free survival.

\section{Author contributions}

Study concept, design, and supervision: Ahmed O. Kaseb. Acquisition of data: Yehia I. Mohamed, Manal M. Hassan, Ahmed O. Kaseb. Analysis and interpretation of data: Yehia I. Mohamed, Manal M. Hassan, Lianchun Xiao, Ahmed O. Kaseb. Drafting of the manuscript: Yehia I. Mohamed, MD, Sunyoung Lee, Lianchun Xiao, Manal M. Hassan, Aliya Qayyum, Rikita Hiatia, Roberto Carmagnani Pestana, Abedul Haque, Bhawana George, Asif Rashid, Dan G. Duda, Hesham Elghazaly, Robert A. Wolff, Jeffrey S. Morris, James Yao, Hesham M. Amin, Ahmed O. Kaseb. Critical revision of the manuscript for important intellectual content: Yehia I. Mohamed, MD, Sunyoung Lee, Lianchun Xiao, Manal M. Hassan, Aliya Qayyum, Rikita Hiatia, Roberto Carmagnani Pestana, Abedul Haque, Bhawana George, Asif Rashid, Dan G. Duda, Hesham Elghazaly, Robert A. Wolff, Jeffrey S. Morris, James Yao, Hesham M. Amin, Ahmed O. Kaseb. Statistical analysis: Yehia I. Mohamed, Manal M. Hassan, Lianchun Xiao, Jeffrey S. Morris. Administrative, technical, or material support: Sunyoung Lee, Lianchun Xiao, Manal M. Hassan, Aliya Qayyum, Rikita Hiatia, Roberto Carmagnani Pestana, Abedul Haque, Bhawana George, Asif Rashid, Dan G. Duda, Robert A. Wolff, Jeffrey S. Morris, James Yao, Hesham M. Amin, Ahmed O. Kaseb.

\section{ACKNOWLEDGMENTS}

Editorial assistance was provided by Laura L. Russel of MD Anderson's Editing Services, Research Medical Library.

\section{CONFLICTS OF INTEREST}

All authors report no commercial associations (e.g., consultancies, stock ownership, equity interests, or patent-licensing arrangements) that might pose a conflict of interest in connection with the submitted article.

\section{FUNDING}

This work was supported by the NIH/NCI under award number P30CA016672 and used the Biostatistics Resource Group. This work was also supported by the NIH through grants CA170035-01 and CA190945-01A1 (AOK), CA106458-01 (MMH), R01 CA151533 (HMA) and by the MD Anderson Cancer Center Sister Institution Network Fund grant (AOK). DGD's research is supported by Dod awards W81XWH-19-1-0284 and W81WXH-19-1-0482.

\section{REFERENCES}

1. Vauthey JN, Dixon E, Abdalla EK, Helton WS, Pawlik TM, Taouli B, Brouquet A, Adams RB, and American HepatoPancreato-Biliary Association, and Society of Surgical Oncology, and Society for Surgery of the Alimentary Tract. Pretreatment assessment of hepatocellular carcinoma: expert consensus statement. HPB (Oxford). 2010; 12:289-99. https://doi.org/10.1111/j.1477-2574.2010.00181.x. [PubMed] 
2. Pugh RN, Murray-Lyon IM, Dawson JL, Pietroni MC, Williams R. Transection of the oesophagus for bleeding oesophageal varices. Br J Surg. 1973; 60:646-9. https://doi. org/10.1002/bjs.1800600817. [PubMed]

3. Llovet JM, Di Bisceglie AM, Bruix J, Kramer BS, Lencioni R, Zhu AX, Sherman M, Schwartz M, Lotze M, Talwalkar J, Gores GJ, and Panel of Experts in HCCDesign Clinical Trials. Design and endpoints of clinical trials in hepatocellular carcinoma. J Natl Cancer Inst. 2008; 100:698-711. https://doi.org/10.1093/jnci/djn134. [PubMed]

4. Wilson SR, Greig P, Kaseb AO. Pretreatment assessment of hepatocellular cancer: expert consensus conference. HPB (Oxford). 2010; 12:300-01. https://doi.org/10.1111/j.14772574.2010.00187.x. [PubMed]

5. Morris JS, Hassan MM, Zohner YE, Wang Z, Xiao L, Rashid A, Haque A, Abdel-Wahab R, Mohamed YI, Ballard KL, Wolff RA, George B, Li L, et al. HepatoScore-14: measures of biological heterogeneity significantly improve prediction of hepatocellular carcinoma risk. Hepatology. 2020 Sep 15. https://doi.org/10.1002/hep.31555. [Epub ahead of print]. [PubMed]

6. Durand F, Valla D. Assessment of the prognosis of cirrhosis: Child-Pugh versus MELD. J Hepatol. 2005 (Suppl); 42:S100 07. https://doi.org/10.1016/j.jhep.2004.11.015. [PubMed]

7. Kim HJ, Lee HW. Important predictor of mortality in patients with end-stage liver disease. Clin Mol Hepatol. 2013; 19:105-15. https://doi.org/10.3350/ cmh.2013.19.2.105. [PubMed]

8. Oellerich M, Burdelski M, Lautz HU, Rodeck B, Duewel J, Schulz M, Schmidt FW, Brodehl J, Pichlmayr R. Assessment of pretransplant prognosis in patients with cirrhosis. Transplantation. 1991; 51:801-06. https://doi. org/10.1097/00007890-199104000-00013. [PubMed]

9. Pessione F, Ramond MJ, Peters L, Pham BN, Batel P, Rueff B, Valla DC. Five-year survival predictive factors in patients with excessive alcohol intake and cirrhosis. Effect of alcoholic hepatitis, smoking and abstinence. Liver Int. 2003; 23:45-53. https://doi.org/10.1034/j.16000676.2003.01804.x. [PubMed]

10. Said A, Williams J, Holden J, Remington P, Gangnon R, Musat A, Lucey MR. Model for end stage liver disease score predicts mortality across a broad spectrum of liver disease. J Hepatol. 2004; 40:897-903. https://doi. org/10.1016/j.jhep.2004.02.010. [PubMed]

11. Testa R, Valente U, Risso D, Caglieris S, Giannini E, Fasoli A, Botta F, Dardano G, Lantieri PB, Celle G. Can the MEGX test and serum bile acids improve the prognostic ability of Child-Pugh's score in liver cirrhosis? Eur J Gastroenterol Hepatol. 1999; 11:559-63. https://doi. org/10.1097/00042737-199905000-00016. [PubMed]

12. Colakoğlu O, Taşkiran B, Colakoğlu G, Kizildağ S, Ari Ozcan F, Unsal B. Serum insulin like growth factor-1 (IGF-1) and insulin like growth factor binding protein-3 (IGFBP-3) levels in liver cirrhosis. Turk J Gastroenterol. 2007; 18:245-49. [PubMed]
13. Buzzelli G, Dattolo P, Pinzani M, Brocchi A, Romano $\mathrm{S}$, Gentilini P. Circulating growth hormone and insulinlike growth factor-I in nonalcoholic liver cirrhosis with or without superimposed hepatocarcinoma: evidence of an altered circadian rhythm. Am J Gastroenterol. 1993; 88:1744-48. [PubMed]

14. Luo SM, Tan WM, Deng WX, Zhuang SM, Luo JW. Expression of albumin, IGF-1, IGFBP-3 in tumor tissues and adjacent non-tumor tissues of hepatocellular carcinoma patients with cirrhosis. World J Gastroenterol. 2005; 11:4272-76. https://doi.org/10.3748/wjg.v11.i27.4272. [PubMed]

15. Stuver SO, Kuper H, Tzonou A, Lagiou P, Spanos E, Hsieh CC, Mantzoros C, Trichopoulos D. Insulin-like growth factor 1 in hepatocellular carcinoma and metastatic liver cancer in men. Int J Cancer. 2000; 87:118-21. https:// doi.org/10.1002/1097-0215(20000701)87:1<118::aidijc17>3.0.co;2-w. [PubMed]

16. Su WW, Lee KT, Yeh YT, Soon MS, Wang CL, Yu ML, Wang SN. Association of circulating insulin-like growth factor 1 with hepatocellular carcinoma: one cross-sectional correlation study. J Clin Lab Anal. 2010; 24:195-200. https://doi.org/10.1002/jcla.20320. [PubMed]

17. García-Galiano D, Sánchez-Garrido MA, Espejo I, Montero JL, Costán G, Marchal T, Membrives A, Gallardo-Valverde JM, Muñoz-Castañeda JR, Arévalo E, De la Mata M, Muntané J. IL-6 and IGF-1 are independent prognostic factors of liver steatosis and non-alcoholic steatohepatitis in morbidly obese patients. Obes Surg. 2007; 17:493-503. https://doi.org/10.1007/s11695-007-9087-1. [PubMed]

18. Völzke H, Nauck M, Rettig R, Dörr M, Higham C, Brabant G, Wallaschofski H. Association between hepatic steatosis and serum IGF1 and IGFBP-3 levels in a population-based sample. Eur J Endocrinol. 2009; 161:705-13. https://doi. org/10.1530/EJE-09-0374. [PubMed]

19. Kaseb AO, Morris JS, Hassan MM, Siddiqui AM, Lin E, Xiao L, Abdalla EK, Vauthey JN, Aloia TA, Krishnan S, Abbruzzese JL. Clinical and prognostic implications of plasma insulin-like growth factor-1 and vascular endothelial growth factor in patients with hepatocellular carcinoma. J Clin Oncol. 2011; 29:3892-99. https://doi.org/10.1200/ JCO.2011.36.0636. [PubMed]

20. Kaseb AO, Abbruzzese JL, Vauthey JN, Aloia TA, Abdalla EK, Hassan MM, Lin E, Xiao L, El-Deeb AS, Rashid A, Morris JS. I-CLIP: improved stratification of advanced hepatocellular carcinoma patients by integrating plasma IGF-1 into CLIP score. Oncology. 2011; 80:373-81. https:// doi.org/10.1159/000329040. [PubMed]

21. Kaseb AO, Xiao L, Hassan MM, Chae YK, Lee JS, Vauthey JN, Krishnan S, Cheung S, Hassabo HM, Aloia T, Conrad C, Curley SA, Vierling JM, et al. Development and validation of insulin-like growth factor-1 score to assess hepatic reserve in hepatocellular carcinoma. J Natl Cancer Inst. 2014; 106:dju088. https://doi.org/10.1093/jnci/dju088. [PubMed] 
22. Lacin S, Yalcin S, Karakas Y, Hassan MM, Amin H, Mohamed YI, Rashid A, Morris JS, Xiao L, Qayyum A, Kaseb AO. Prognostic Significance of Serum Insulin-Like Growth Factor-1 in Hepatocellular Cancer Patients: A Validation Study. J Hepatocell Carcinoma. 2020; 7:143-53. https://doi.org/10.2147/JHC.S258930. [PubMed]

23. Abdel-Wahab R, Shehata S, Hassan MM, Xiao L, Lee JS, Cheung S, Essa HH, Hassabo HM, Shalaby AS, Mosad E, Raghav K, Rashid A, Wolff RA, et al. Validation of an IGF-CTP scoring system for assessing hepatic reserve in Egyptian patients with hepatocellular carcinoma. Oncotarget. 2015; 6:21193-207. https://doi.org/10.18632/ oncotarget.4176. [PubMed]

24. Abdel-Wahab R, Lacin S, Shalaby AS, Hassan M, Xiao L, Morris J, Amin HM, Kaseb AO. Integrating insulin-like growth factor-1 (IGF-1) score into Barcelona Clinic Liver Cancer (BCLC) and Cancer of the Liver Italian Program (CLIP) scores. J Clin Oncol. 2016; 34:4081. https://doi. org/10.1200/JCO.2016.34.15_suppl.4081.

25. Abdel-Wahab R, Hassan MM, George B, Carmagnani Pestana R, Xiao L, Lacin S, Yalcin S, Shalaby AS, Al-Shamsi HO, Raghav K, Wolff RA, Yao JC, Girard L, et al. Impact of Integrating Insulin-Like Growth Factor 1 Levels into Model for End-Stage Liver Disease Score for Survival Prediction in Hepatocellular Carcinoma Patients. Oncology. 2020; 98:83646. https://doi.org/10.1159/000502482. [PubMed]
26. Cheng AL, Kang YK, Chen Z, Tsao CJ, Qin S, Kim JS, Luo R, Feng J, Ye S, Yang TS, Xu J, Sun Y, Liang H, et al. Efficacy and safety of sorafenib in patients in the AsiaPacific region with advanced hepatocellular carcinoma: a phase III randomised, double-blind, placebo-controlled trial. Lancet Oncol. 2009; 10:25-34. https://doi.org/10.1016/ S1470-2045(08)70285-7. [PubMed]

27. Llovet JM, Ricci S, Mazzaferro V, Hilgard P, Gane E, Blanc JF, de Oliveira AC, Santoro A, Raoul JL, Forner A, Schwartz M, Porta C, Zeuzem S, et al, and SHARP Investigators Study Group. Sorafenib in advanced hepatocellular carcinoma. N Engl J Med. 2008; 359:37890. https://doi.org/10.1056/NEJMoa0708857. [PubMed]

28. El-Serag HB, Rudolph KL. Hepatocellular carcinoma: epidemiology and molecular carcinogenesis. Gastroenterology. 2007; 132:2557-76. https://doi. org/10.1053/j.gastro.2007.04.061. [PubMed]

29. Harrell FE Jr, Lee KL, Mark DB. Multivariable prognostic models: issues in developing models, evaluating assumptions and adequacy, and measuring and reducing errors. Stat Med. 1996; 15:361-87. https://doi. org/10.1002/(sici)1097-0258(19960229)15:4<361::Aidsim168>3.0.Co;2-4. [PubMed] 